\title{
Identification of Anisotropic Yield Criterion Parameters from a Single Biaxial Tensile Test
}

\author{
ZHANG Shunying ${ }^{1, a}$, LEOTOING Lionel ${ }^{1, b}$, GUINES Dominique ${ }^{1, c^{*}}$ and \\ THUILLIER Sandrine $e^{2, d}$ \\ ${ }^{1}$ LGCGM, INSA de Rennes, CS70839 - 35708 Rennes Cedex 7, France \\ ${ }^{2}$ LIMATB, Univ. Bretagne-Sud, F-56100 Lorient, France \\ ashunying.zhang@insa-rennes.fr, blionel.leotoing@insa-rennes.fr, \\ cdominique.guines@insa-rennes.fr, 'sandrine.thuillier@univ-ubs.fr
}

Keywords: Plastic anisotropy, Yield criterion, Biaxial test, Material parameter identification.

\begin{abstract}
The present work deals with the calibration strategy of yield functions used to describe the plastic anisotropic behavior of metallic sheets. In this paper, Bron and Besson yield criterion is used to model the plastic anisotropic behavior of AA5086 sheets. This yield model is flexible enough since the anisotropy is represented by 12 parameters ( 4 isotropic parameters and 8 anisotropic parameters in plane stress condition) in the form of two linear fourth order transformation tensors. The parameters of this anisotropic yield model have been identified from a single dedicated cross biaxial tensile test. It is shown, from finite element simulations, that the strain distribution in the center of the cruciform specimen is significantly dependent on the yield criterion. Moreover, this cross biaxial test involves a large range of strain paths in the center of the specimen. The calibration stage is performed by means of an optimization procedure minimizing the gap between experimental and numerical values of the principal strains along a specified path in the gauge area of the cruciform specimen. It is shown that the material parameters of Bron and Besson anisotropic yield model can be determined accurately by a unique biaxial tensile test.
\end{abstract}

\section{Introduction}

Sheet metals usually exhibit a plastic anisotropy due to previous thermo-mechanical processes like rolling and annealing. To optimize the numerical simulation of these forming processes, an accurate description of the plastic behavior is required. Within a phenomenological description of the mechanical behavior of sheet metals, yield functions and especially anisotropic ones are used to represent the initial anisotropy of the material. Many anisotropic yield models were proposed to describe the initial anisotropy, e.g. a thorough review of these models is presented in [1]. The initial anisotropy description, coupled with hardening evolution, can lead to a good representation of the mechanical behavior over a large strain range, as shown in [2]. An alternative consists in taking into account anisotropy evolution, as proposed in [3]. To consider the plastic strain-induced anisotropy, Zang and Lee [4] carried out the eigen decompositions of the linear transformation tensors of Yld2000-2d yield model at different equivalent plastic strains.

Yield functions can involve a high number of material parameters. The calibration of these parameters requires usually several mechanical tests with different loading paths. To guarantee the relevance of the parameter set, the number of experimental data should not be lower than the number of material parameters considered in the identification process. The relevance of the yield contour is improved when increasing the number of sampling points, demanding an increase of experimental information. However, from an economical point of view, the number of tests should be as small as possible. It has been proposed in [1] that the following experimental data is required at least: three yield stresses (e.g. $\sigma_{0}, \sigma_{45}$ and $\sigma_{90}$ ) and three anisotropic coefficients (e.g. $\mathrm{r}_{0}, \mathrm{r}_{45}$ 
and $r_{90}$ ) obtained from uniaxial tensile tests in different orientations to the rolling direction (RD); an equi-biaxial yield stress $\left(\sigma_{b}\right)$ and a biaxial coefficient $\left(r_{b}\right)$ from biaxial tensile test, usually hydraulic bulge test. As mentioned above, most of the previous works proposed identification based on the initial values of these data, measured at the elasto-plastic transition.

However, $\mathrm{Hu}$ [5] pointed out that the initial yield stresses were difficult to determine accurately since there exist several definition of initial yielding. Some works investigated the identification of material parameters considering not only the initial values but also values recorded at higher strains [6,7]. Another approach without considering initial yield stress values consists in parameter identification over the temporal evolution of experimental data. Zang et al [2] considered a combination of stress level in uniaxial tension, equi-biaxial tension and simple shear, both monotonic and Bauschinger tests, to identify material parameters of Bron and Besson yield function. Bron and Besson [8] also proposed a similar identification strategy with the temporal evolution of stress levels in tensile tests, both on straight and U-notched samples. It can be concluded that due to the dispersion on initial yield stresses, as well as the evolution of anisotropy with strain, considering only initial yield stresses does not give an accurate description of the mechanical behavior.

Recently, some works have been focused on parameter identification of yield functions from the biaxial tensile test. Green et al [9] have performed cross biaxial tests with seven different proportional strain paths, in order to identify the parameters of several yield functions. The authors adjusted the parameters with an iterative procedure to optimize the predicted strength level of two arms of the cruciform sample. Teaca et al [10] proposed the identification Ferron, Makkouk and Morreale (FMM) yield function parameters [11] by combining results of uniaxial tensile tests and cross biaxial test. However, only two parameters of the yield model were calibrated from the strain distribution in the central part of the cruciform specimen. The field measurement of the strain level was also used by Prates et al. [12] to identify Hill 1948 coefficients. Up to now and to the authors' knowledge, there is no published work that concerns the parameter identification of a complex yield model with only one cross biaxial tensile test.

In the present article, a biaxial tensile test is performed on a cruciform specimen in order to investigate the plastic anisotropy of AA5086 sheets. Bron and Besson yield model, chosen to represent this anisotropic behavior, is expressed with two linear fourth order transformation tensors involving 12 material parameters; i.e. 4 isotropic parameters and 8 anisotropic parameters in plane stress condition. Experimental results show that the strain distribution in the central area of the proposed cross flat specimen is significantly sensitive to the yield criterion. Consequently, an identification procedure of all Bron and Besson parameters, based on a unique biaxial tensile test, is led. Comparisons between experimental and numerical results of principal strains along a specified path defined in the gauge area of the cruciform specimen are presented. It is shown that the cross biaxial test involves a large range of strain paths, though the maximum strain is limited. Finally, the yield function identified by the proposed method is compared to the one obtained from a classical identification approach.

\section{Biaxial Tensile Test with Cruciform Specimen}

A cruciform specimen shape has been designed and is shown in Fig. 1. Experiments on a servohydraulic testing machine have been performed [13] with a constant velocity of $1 \mathrm{~mm} / \mathrm{s}$ imposed on the four arms of the cruciform specimen. The AA5086 sheet has a thickness of $2 \mathrm{~mm}$ and the orientation of the rolling direction corresponds to the $X$ axis. The Fig. 2 shows the evolution of the loads $F_{x}$ and $F_{y}$ recorded along the two arms. It can be seen that due to the anisotropy, a significant difference is observed.

Images of the central area of the specimen were recorded with a high resolution camera and a digital image correlation (DIC) software CORRELA 2D (developed by LMS at the University of Poitiers) was used to compute the in-plane strain components. As shown in Fig. 3, a central square 
area of approximately $25 \times 25 \mathrm{~mm}^{2}$ was selected, leading to a total number of about 1600 points where the strains are calculated.

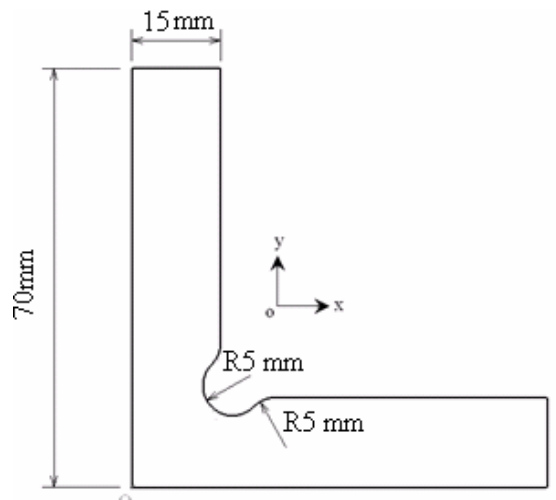

Fig. 1: Geometry of quarter cruciform specimen

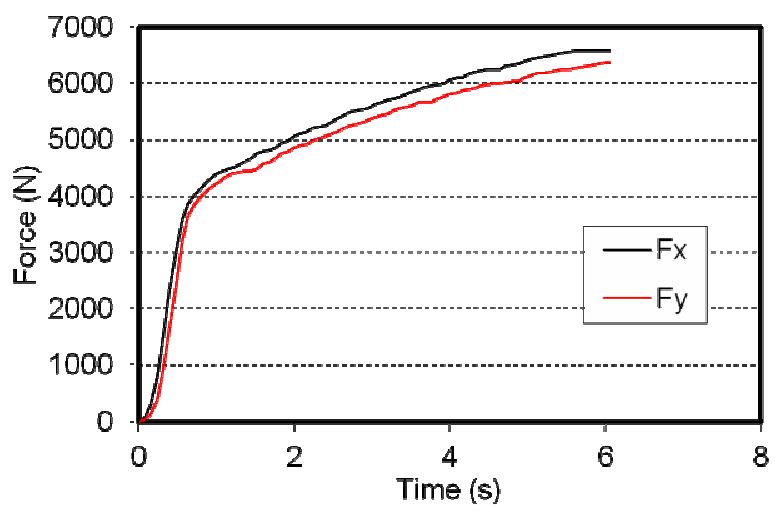

Fig. 2 Evolution of loads on two arms

In-plane major strain $\varepsilon_{1}$ and minor strain $\varepsilon_{2}$ were output at these material points and the strain path, characterized by the ratio $\varepsilon_{2} / \varepsilon_{1}$, was analyzed at time $t=6$.0s for a rupture recorded at time $\mathrm{t}=6.048 \mathrm{~s}$. Such a distribution is presented in Fig. 4. There is a nearly equi-biaxial strain state in the central area. It then changes gradually to nearly uniaxial tensile stress state at the corner.

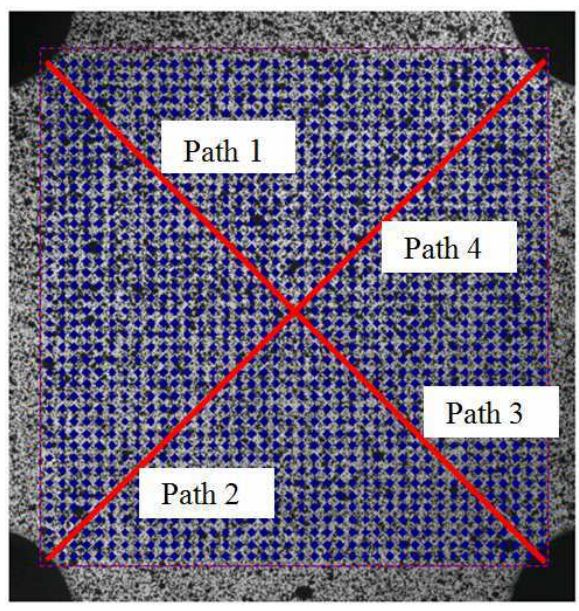

Fig. 3: Analyzed section (in blue, $25 \times 25 \mathrm{~mm}^{2}$ ) and visualization of the 4 diagonal paths.

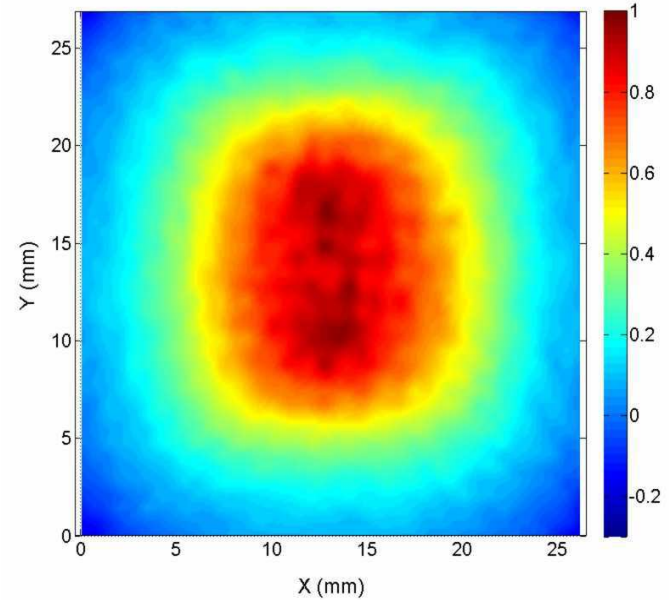

Fig. 4 Strain path ratio distribution $\varepsilon_{2} / \varepsilon_{1}$ in the analyzed central section

The maximum and minimum principal strains along four diagonal paths indicated in Fig. 3 have been compared in Figs. 5 and 6 respectively. The results obtained for the four paths are similar, whatever the selected path. A slight discrepancy is recorded near the free edge of the sample, the maximum relative gap being $1.7 \%$ for the major strain and $0.6 \%$ for the minor strain. An average value, both for minor and major strains, was then calculated over the four paths. This average is used in the following parts for the comparison with finite element (FE) simulation and identification procedure. 


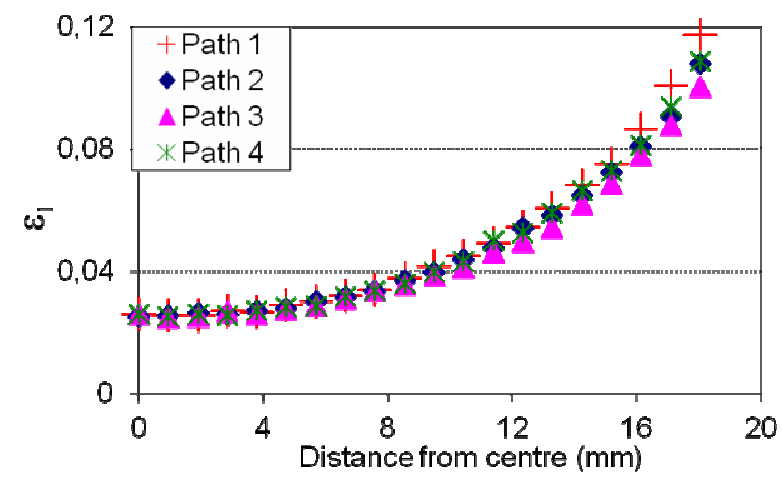

Fig. 5 Major principal strain along the four diagonal paths

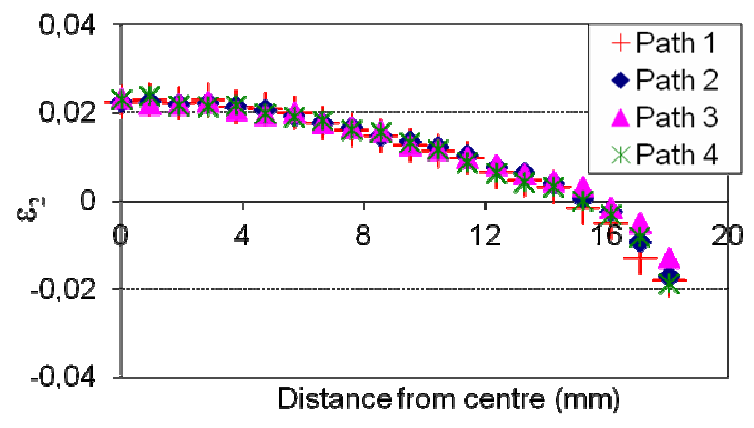

Fig. 6 Minor principal strain along the four diagonal paths

\section{Material Model}

Bron and Besson proposed a yield function involving 16 parameters under the form [8]:

$$
\psi=\left(\sum_{\mathrm{k}=1}^{2} \alpha^{\mathrm{k}}\left(\bar{\sigma}^{\mathrm{k}}\right)^{\mathrm{a}}\right)^{1 / \mathrm{a}}=\bar{\sigma}=\mathrm{Y}_{0}
$$

$\alpha^{k}$ are positive coefficients, the sum of which is equal to $1 . \bar{\sigma}$ is the equivalent stress and $Y_{0}$ a reference yield stress of the material. $\bar{\sigma}^{\mathrm{k}}, \mathrm{k}=1,2$ are expressed in the form:

$$
\begin{aligned}
& \bar{\sigma}^{1}=\left(\frac{1}{2}\left(\left|S_{2}^{1}-S_{3}^{1}\right|^{b_{1}}+\left|S_{3}^{1}-S_{1}^{1}\right|^{b_{1}}+\left|S_{2}^{1}-S_{1}^{1}\right|^{b_{1}}\right)\right)^{1 / b_{1}} \\
& \bar{\sigma}^{2}=\left(\frac{3 b_{2}}{2^{b_{2}}+2}\left(\left|S_{1}^{2}\right|^{b_{2}}+\left|S_{2}^{2}\right|^{b_{2}}+\left|S_{3}^{2}\right|^{b_{2}}\right)\right)^{1 / b_{2}}
\end{aligned}
$$

$\mathrm{a}, \mathrm{b}_{1}, \mathrm{~b}_{2}$ and $\alpha^{1}\left(\alpha^{2}=1-\alpha^{1}\right)$ are four isotropic parameters which define the shape of the yield surface. $S_{i}^{k}$ are the principal values of the transformed stress deviators $s_{i j}^{\prime k}$ defined by:

$\mathrm{s}_{\mathrm{ij}}^{\prime \mathrm{k}}=\mathrm{L}^{\mathrm{k}} \sigma_{\mathrm{ij}}$

with $\mathrm{L}^{\mathrm{k}}=\left(\begin{array}{cccccc}\frac{\mathrm{c}_{2}^{\mathrm{k}}+\mathrm{c}_{3}^{\mathrm{k}}}{3} & -\frac{\mathrm{c}_{3}^{\mathrm{k}}}{3} & -\frac{\mathrm{c}_{2}^{\mathrm{k}}}{3} & 0 & 0 & 0 \\ -\frac{\mathrm{c}_{3}^{\mathrm{k}}}{3} & \frac{\mathrm{c}_{1}^{\mathrm{k}}+\mathrm{c}_{3}^{\mathrm{k}}}{3} & -\frac{\mathrm{c}_{1}^{\mathrm{k}}}{3} & 0 & 0 & 0 \\ -\frac{\mathrm{c}_{2}^{\mathrm{k}}}{3} & -\frac{\mathrm{c}_{1}^{\mathrm{k}}}{3} & \frac{\mathrm{c}_{1}^{\mathrm{k}}+\mathrm{c}_{2}^{\mathrm{k}}}{3} & 0 & 0 & 0 \\ 0 & 0 & 0 & \mathrm{c}_{4}^{\mathrm{k}} & 0 & 0 \\ 0 & 0 & 0 & 0 & \mathrm{c}_{5}^{\mathrm{k}} & 0 \\ 0 & 0 & 0 & 0 & 0 & \mathrm{c}_{6}^{\mathrm{k}}\end{array}\right)$

Where $c_{i}^{k}$ are 12 parameters, which are related to the material anisotropy. In the case of plane stress condition, the anisotropic parameter number reduces to 8 with $c_{5}^{\mathrm{k}}=\mathrm{c}_{6}^{\mathrm{k}}=1$.

\section{Parameter Identification of Bron and Besson Yield Function}

From experimental results of the biaxial tensile test presented above, an identification procedure of Bron and Besson yield function parameters is proposed. It consists in the minimization of the gap between the experimental and calculated evolutions of major and minor strains along the diagonal 
path defined in the central area of the specimen (Fig. 3). The calculated values are obtained from a FE numerical model of the biaxial tensile test. All simulations have been carried out with the commercial software ABAQUS. Bron and Besson yield function is implemented through the UMAT user subroutine. It should be emphasized that, as a first step, only anisotropy is dealt with. Hardening of the material is modeled by isotropic hardening identified from a tensile test in the rolling direction. The hardening law adopted here is:

$$
\bar{\sigma}=\sigma_{0}+\mathrm{A}\left(1-\mathrm{e}^{-\mathrm{B} \overline{\mathrm{p}}_{\mathrm{p}}}\right)
$$

where $\sigma_{0}=146 \mathrm{MPa}$ is the initial yield strength. $\mathrm{A}=217.6 \mathrm{MPa}$ and $\mathrm{B}=10.9$ are material parameters.

Due to the symmetry of the problem, only a quarter of the specimen is modeled (Fig. 7). Experimental forces $F_{x}$ and $F_{y}$ given in Fig. 2 are imposed on the two arms of the cruciform specimen during the simulation process. Four node shell elements were used for the mesh, with a minimum size of $1 \mathrm{~mm}$. Influence of the mesh size was investigated, in particular its influence on the major and minor strains, and stable predictions were obtained with the selected mesh.

The cost function evaluates the difference between the experimental and numerical principal strains and is defined by:

$$
\delta_{\mathrm{b}}\left(\mathrm{Y}_{0} / \sigma_{0}, \mathrm{a}, \mathrm{b}^{1}, \mathrm{~b}^{2}, \alpha_{1}, \mathrm{c}_{\mathrm{i}}^{\mathrm{k}}\right)=\sum_{\mathrm{i}=1}^{\mathrm{p}}\left(\frac{\varepsilon_{1}^{\mathrm{EFi}}-\varepsilon_{1}^{\mathrm{i}}}{\varepsilon_{1}^{\mathrm{i}}}\right)^{2}+\sum_{\mathrm{i}=1}^{\mathrm{p}}\left(\frac{\varepsilon_{2}^{\mathrm{EFi}}-\varepsilon_{2}^{\mathrm{i}}}{\varepsilon_{2}^{\mathrm{i}}}\right)^{2}
$$

where experimental values $\varepsilon_{1}$ and $\varepsilon_{2}$ are compared to the numerical values $\varepsilon_{1}^{\mathrm{EF}}$ and $\varepsilon_{2}^{\mathrm{EF}}$. The index $\mathrm{p}$ in Eq. (6) stands for the number of points along the diagonal path. As the nodes used to output the strain components are different in the model and in the experiments, a linear interpolation of the experimental signals was performed. During the optimization process, the principal strain field at time $\mathrm{t}=6.0 \mathrm{~s}$ is considered both in the experiments and in the numerical simulation. The identification is realized with the commercial software modeFRONTIER $\AA$, which is an integration platform for multi-objective optimization. It provides a coupling with the FE software ABAQUS. The algorithm SIMPLEX is preferred in the identification process. In a situation with finite element inverse integration, which takes long time for each calculation, the algorithm SIMPLEX is well adapted.

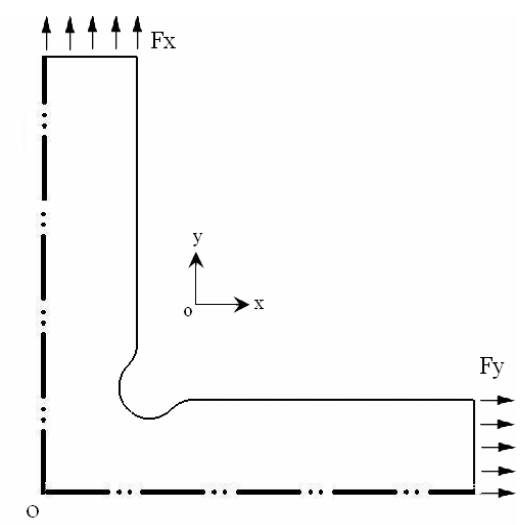

Fig. 7: FE boundary conditions. Dashed lines correspond to symmetric conditions. 


\section{Results}

Table 1 gives the variation range and the initial value of each parameter and values of identified parameters of Bron and Besson yield model.

Table 1. Parameters of Bron and Besson yield function identified by biaxial test

\begin{tabular}{|c|c|c|c|c|c|c|c|c|c|c|c|c|}
\hline $\begin{array}{l}\mathrm{Y}_{0} / \sigma_{0} \\
{[\mathrm{MPa}]}\end{array}$ & $\alpha_{1}$ & $\mathrm{a}$ & $b_{1}$ & $\mathrm{~b}_{2}$ & $c_{1}^{1}$ & $c_{2}^{1}$ & $c_{3}^{1}$ & $c_{4}^{1}$ & $c_{1}^{2}$ & $c_{2}^{2}$ & $c_{3}^{2}$ & $c_{4}^{2}$ \\
\hline $\begin{array}{c}1 \\
0.8 \sim 1.2\end{array}$ & $\begin{array}{c}0.5 \\
0.1 \sim 0.9\end{array}$ & $\begin{array}{c}6 \\
0 \sim 12\end{array}$ & $\begin{array}{c}10 \\
0 \sim 20\end{array}$ & $\begin{array}{c}10 \\
0 \sim 20\end{array}$ & $\begin{array}{c}0.5 \\
-1.2 \sim 2.2\end{array}$ & $\begin{array}{c}0.5 \\
-1.2 \sim 2.2\end{array}$ & $\begin{array}{c}0.5 \\
-1.2 \sim 2.2\end{array}$ & $\begin{array}{c}0.5 \\
-1.2 \sim 2.2\end{array}$ & $\begin{array}{c}0.5 \\
-1.2 \sim 2.2\end{array}$ & $\begin{array}{c}0.5 \\
-1.2 \sim 2.2\end{array}$ & $\begin{array}{c}0.5 \\
-1.2 \sim 2.2\end{array}$ & $\begin{array}{c}0.5 \\
-1.2 \sim 2.2\end{array}$ \\
\hline 0.862 & 0.72 & 0.16 & 13.00 & 8.41 & 1.06 & 1.10 & 0.82 & 0.95 & 0.75 & 0.47 & 0.78 & 0.62 \\
\hline
\end{tabular}

Fig. 8 gives the equivalent plastic strain distribution in the central area of the specimen at $\mathrm{t}=6.0 \mathrm{~s}$ with the identified Bron and Besson model.

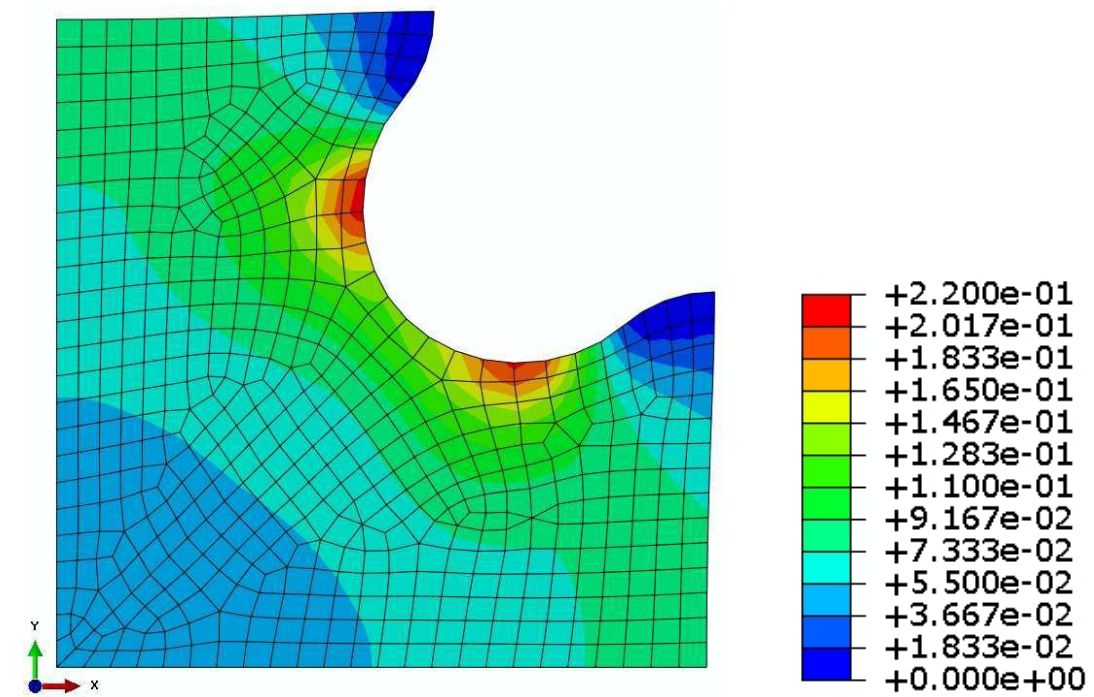

Fig. 8: Equivalent plastic strain distribution in the central area of the specimen

Fig. 9 shows the predicted and experimental major and minor strain evolution along a diagonal path. According to the figure, there is a very good agreement between experiments and numerical simulation. The strain path ratio along the diagonal direction has been compared with the experimental one in Fig. 10. Bron and Besson model gives a slight underestimation at the beginning of the curve (central area of the cruciform specimen). However, farther from the center, the prediction is rather close to the experiments.

Fig. 11 gives a comparison between two yield contours calculated with parameters of Bron and Besson model obtained either from the biaxial test or from conventional tests [14]. There is only a small difference between these two contours, mainly near the plane strain state. 


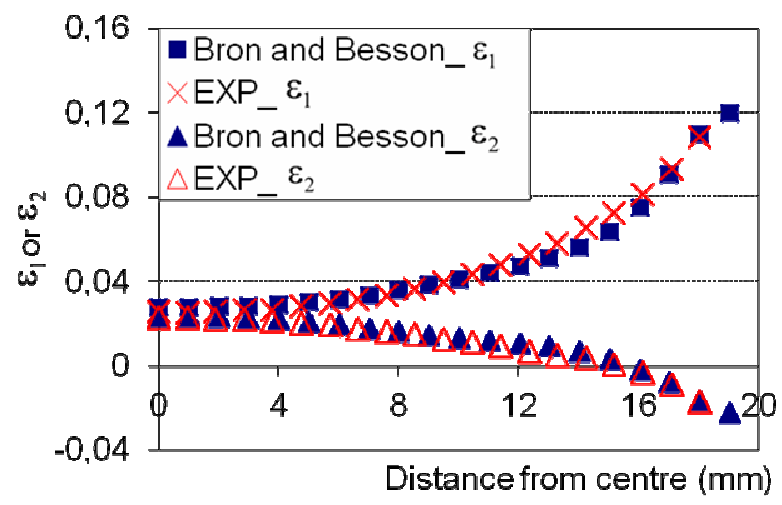

Fig. 9 Principal strains

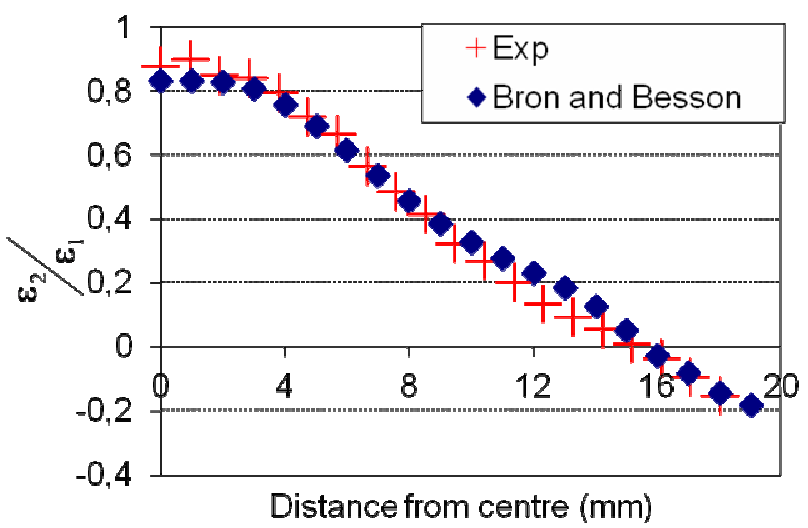

Fig. 10 Strain path ratio

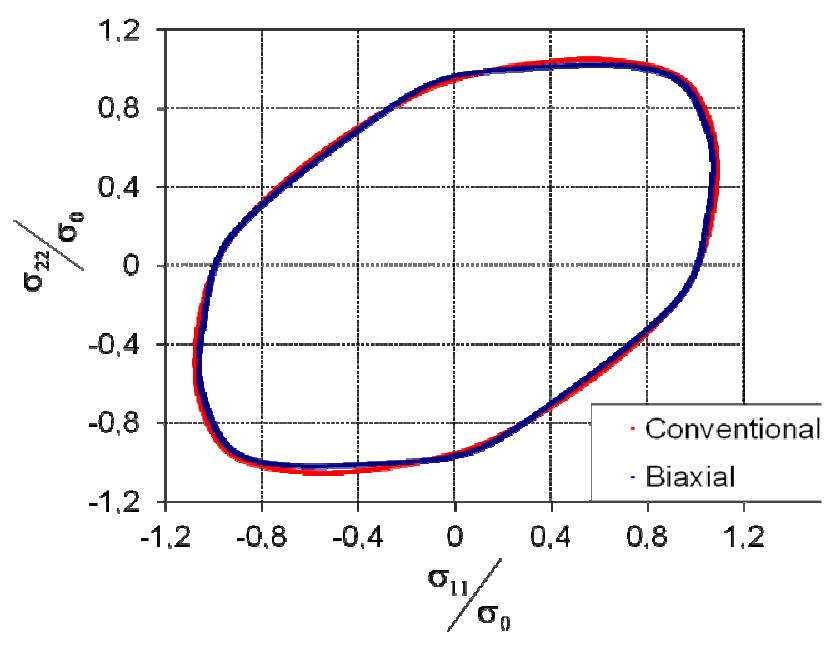

Fig. 11 Comparison of two yield contours

\section{Conclusion}

To simplify both the obtaining of the experimental database and the calibration of the yield function parameters, an identification method based on data obtained from a single cross biaxial tensile test was investigated.

Bron and Besson yield model has been applied to investigate the anisotropic behavior of material AA5086. A cost function, based on the minimization of the gap between the experimental and calculated evolutions of major and minor strains along a diagonal path in the central area of the specimen, has been defined. From the proposed identification method, all the 13 parameters of Bron and Besson yield function have been determined with only one biaxial tensile test. Good agreement between yield contours obtained from an identification process based on the biaxial test and from a classical method of calibration based on conventional tests validates the proposed method.

\section{References}

[1] D. Banabic, Sheet Metal Forming Processes: Constitutive Modeling and Numerical Simulation, Springer. 2010.

[2] S. L. Zang, S. Thuillier, A. Le Port, and P. Y. Manach, Prediction of anisotropy and hardening for metallic sheets in tension, simple shear and biaxial tension, Int. J. Mech. Sci. 53 (2011) 338-347.

[3] Y. S. Suh, F. I. Saunders, and R. H. Wagoner, Anisotropic yield functions with plasticstrain-induced anisotropy, Int. J. Plast. 12 (1996) 417-438. 
[4] S. Zang and M. Lee, A General Yield Function within the Framework of Linear Transformations of Stress Tensors for the Description of Plastic - strain - induced Anisotropy, AIP Conf. Proc. 1383 (2011) 63-70.

[5] W. Hu, Constitutive modeling of orthotropic sheet metals by presenting hardening-induced anisotropy, Int. J. Plast. 23 (2007) 620-639.

[6] P. D. Barros, M. C. Oliveira, J. L. Alves, and L. F. Menezes, Earing Prediction in Drawing and Ironing Processes Using an Advanced Yield Criterion, Key Eng. Mater. 554-557 (2013) 22662276.

[7] H. Wang, M. Wan, X. Wu, and Y. Yan, The equivalent plastic strain-dependent Yld2000-2d yield function and the experimental verification, Comput. Mater. Sci. 47 (2009) 12-22.

[8] F. Bron and J. Besson, A yield function for anisotropic materials Application to aluminum alloys, Int. J. Plast. 20 (2004) 937-963.

[9] D. E. Green, K. W. Neale, S. R. MacEwen, A. Makinde, and R. Perrin, Experimental investigation of the biaxial behaviour of an aluminum sheet, Int. J. Plast. 20 (2004) 1677-1706.

[10] M. Teaca, I. Charpentier, M. Martiny, and G. Ferron, Identification of sheet metal plastic anisotropy using heterogeneous biaxial tensile tests, Int. J. Mech. Sci. 52 (2010) 572-580.

[11] G. Ferron, R. Makkouk, and J. Morreale, A parametric description of orthotropic plasticity in metal sheets, Int. J. Plast. 10 (1994) 431-449.

[12] P. A. Prates, J. V. Fernandes, M. C. Oliveira, N. A. Sakharova, and L. F. Menezes, On the characterization of the plastic anisotropy in orthotropic sheet metals with a cruciform biaxial test, IOP Conf. Ser. Mater. Sci. Eng. 10 (2010) 012142.

[13] I. Zidane, D. Guines, L. Léotoing, and E. Ragneau, Development of an in-plane biaxial test for forming limit curve (FLC) characterization of metallic sheets, Meas. Sci. Technol. 21 (2010) 055701.

[14] S. Zhang, L. Léotoing, D. Guines, S. Thuillier, Calibration of material parameters of anisotropic yield criterion with conventional tests and biaxial test, Proceedings Esaform 2013. 